\title{
Forming and ending marital or cohabiting relationships in a Danish population-based cohort of individuals with neurofibromatosis 1
}

\author{
Trille Kristina Kjaer ${ }^{1} \cdot$ Elisabeth Wreford Andersen ${ }^{2} \cdot$ Maja Olsen $^{1} \cdot$ Line Kenborg $^{3} \cdot$ Pernille Envold Bidstrup $^{1}$. \\ Karoline Doser ${ }^{1,3}$. Hanne Hove ${ }^{4,5}$. John R. Østergaard ${ }^{6}$. Christoffer Johansen ${ }^{1,7}$. Sven Asger Sørensen ${ }^{8}$. \\ John J. Mulvihill ${ }^{9}$ Jeanette F. Winther ${ }^{3,10} \cdot$ Susanne Oksbjerg Dalton ${ }^{1,11}$
}

Received: 26 November 2019 / Revised: 22 April 2020 / Accepted: 28 April 2020 / Published online: 18 May 2020

(c) The Author(s), under exclusive licence to European Society of Human Genetics 2020

\begin{abstract}
Individuals with neurofibromatosis 1 (NF1) may have problems in managing the transition between childhood and adulthood, such as forming a relationship or finding a partner. We aimed to determine the association between NF1 and forming and ending marital or cohabiting relationships by comparing a large Danish population of adults with NF1 with population comparisons. In this population-based cohort study, we compared a population of Danish adults who were hospitalized for or with complications to prior diagnosed NF1 $(n=787)$ with population comparisons matched on gender and birth year $(n=7787)$ through nationwide registries with annually updated information on marriage and cohabitation. Discrete-time survival models were used to estimate hazard ratios (HRs) and 95\% confidence intervals (CIs) for the formation and termination of relationships, with adjustment for birth year, gender, and somatic and psychiatric comorbidities at entry. Individuals with NF1 were significantly less likely to form a relationship (HR $=0.65$; 95\% CI: $0.58-0.73$ ), with the lowest association for individuals $\geq 33$ years (HR 0.40; 95\% CI: 0.25-0.63) and the highest for those aged 18-20 years (HR 0.82; 95\% CI: 0.70-0.96). No significant difference was found for ending relationships (HR 1.00; 95\% CI: 0.86-1.16). In conclusion, individuals who were hospitalized for NF1 are less likely to engage in marital or cohabiting relationships than population comparisons and are older when they form their first relationship. Once a relationship has been established, however, couples with a NF1-individual are not at greater risk of ending the relationship.
\end{abstract}

\section{Introduction}

Neurofibromatosis 1 (NF1) is characterized by a wide range of somatic complications (nonpsychiatric) [1] as well as social and behavioral problems [2]. Previous studies, mainly

Trille Kristina Kjaer

trille@cancer.dk

1 Survivorship and Inequality in Cancer, Danish Cancer Society Research Center, Copenhagen, Denmark

2 Statistics and Pharmacoepidemiology, Danish Cancer Society Research Center, Copenhagen, Denmark

3 Childhood Cancer Research Group, Danish Cancer Society Research Center, Copenhagen, Denmark

4 The RAREDIS Database, Copenhagen University Hospital at Rigshospitalet, Copenhagen, Denmark

5 Center for Rare Diseases, Copenhagen University Hospital at Rigshospitalet, Copenhagen, Denmark with children, suggest that individuals with NF1 are at increased risk of cognitive dysfunction, learning disabilities, attention-deficit-hyperactivity disorder (ADHD), impaired executive functioning, and autism spectrum disorder [2, 3]. The disease is highly unpredictable and often progressive,

6 Centre for Rare Diseases, Paediatrics and Adolescent Medicine, Aarhus University Hospital, Aarhus, Denmark

7 Oncology Clinic, Finsen Center, Copenhagen University Hospital at Rigshospitalet, Copenhagen, Denmark

8 Department of Neurogenetics, Institute of Cellular and Molecular medicine, The Panum Institute, University of Copenhagen, Copenhagen, Denmark

9 University of Oklahoma Health Sciences Center, Oklahoma City, OK, USA

10 Department of Clinical Medicine, Faculty of Health, Aarhus University, Aarhus, Denmark

11 Department of Clinical Oncology and Palliative Care, Zealand University Hospital, Næstved, Denmark 
has a complex set of clinical manifestations, variation in severity and visibility, and reduced life expectancy, due mainly to malignancies [4].

Few studies have addressed the social, emotional, and psychological impact of NF1 in adults [5]. The poor social relationships that some children with NF1 experience during childhood may continue into adulthood [5]. Adolescents and young adults with NF1 are more likely to be lonely than their unaffected siblings [6] and frequently struggle to maintain interpersonal relationships [7], which may be attributable to reduced ability to engage in prosocial behavior rather than antisocial behavior per se [5]. Many adolescents and adults with NF1 report less self-confidence than the general population $[8,9]$ and are more likely to experience symptoms of depression and anxiety [10]. The ability to form and maintain intimate relationships by building skills and experience in initiating sexual activity, entering a long-term union, or starting a family is an important part of the development and transition to adulthood and may affect long-term health and well-being [11]. We hypothesized that individuals with NF1 have problems in managing the transition between childhood and adulthood, such as forming an intimate relationship or finding a partner. The aim of this study was to investigate the association between NF1 and marital or cohabiting relationships by comparing a large Danish population of adults with NF1 with population comparisons matched on gender and year of birth.

\section{Materials and methods}

\section{Study population}

The population-based NF1 cohort has been described previously [12]. In brief, the study cohort consisted of (i) a register-based cohort of all individuals hospitalized for or with complications to prior diagnosed NF1 identified in the Danish National Patient Register (DNPR) $(n=2420)$ and (ii) a clinical NF1 cohort of 636 individuals affiliated with one of two national centers for rare diseases, at Copenhagen University Hospital, Rigshospitalet and at Aarhus University Hospital [13]. Of the 636 individuals in the clinical cohort, 95 individuals were not registered in the DNPR. In total 2515 individuals were included in main cohort.

The DNPR records all inpatient hospitalizations since 1977 and outpatient hospital contacts for somatic diseases since 1995, with medically verified diagnoses coded according to the International Classification of Diseases (ICD), revision 8 and ICD-10 [14]. Although NF1 and NF2 are not clearly differentiated in the DNPR, we excluded 59 individuals with presumed NF2 according to the original notification forms to the Danish Cancer Registry or discharge history in the DNPR. The unique Danish personal identification number assigned to all Danish residents at the time of birth was used to ensure accurate linkage of information among all Danish registries.

The inclusion criteria for the present study cohort were registration for NF1 in Denmark between 1977 (start of DNPR) and 2015, born in Denmark between 1971 and 1997, and being alive and living in Denmark the year before their 18th birthday. Study participants were followed from the age of 18. Only those who had not been registered as in a marriage or cohabiting relationship before the age of 18 were included. After exclusions, a total of 787 individuals with NF1 were included for the present study cohort.

\section{Control cohort}

For each individual with NF1, ten population comparisons from the nationwide Danish Civil Registration System, which contains vital and marital status for all Danish residents since 1968 [15], were matched on gender and month and year of birth, resulting in 7787 population comparisons.

\section{Information on forming and ending marriages or cohabiting relationships}

All individuals with NF1 and corresponding population comparison persons were linked to the Household and Family Statistics, a national database provided by Statistics Denmark, which contains annual data on individual marital and cohabitation relationships since 1986 [16]. The core variables for the database are obtained once a year (January 1st) by linkage to the Danish Civil Registration System [15]. The Database categorizes all Danish couples into one of three family types as follows: married, in a registered partnership (marriage between two people of the same sex), or cohabiting (not married, with or without one or more common children). Cohabitation is defined as two unmarried persons of the opposite sex over the age of 16 , with a maximum age difference of 15 years, who were not from the same family of origin, who live at the same address with no other adults in residence (with or without one or more common children). Thus, for this study, the term "marital or cohabiting relationships" included all three family types. Couples in a relationship but not married or living together were not included in this definition. Ending a relationship was defined as divorce for married couples or registered partners and as no longer sharing the same address for cohabiting couples.

\section{NF1-related morbidity}

Information on somatic and psychiatric NF1-related morbidity was obtained from the DNPR and the Danish Psychiatry Research Register [17], respectively. Only somatic 
diseases that have been shown to be most prevalent in individuals with NF1 [1] were included; i.e., cancer, bone diseases, diseases of the nervous system, diseases of the circulatory system, and other somatic disorders. Psychiatric disorders were grouped into anxiety, depression, ADHD, autism, and other psychiatric disorders [12]. (For ICD-10 codes, see Table 1.)

\section{Statistical analysis}

As updated information on relationship status was available only once a year, on January 1st, discrete-time survival models were used to estimate the hazard ratios (HRs) and 95\% confidence intervals (CIs) for the formation and termination of relationships according to NF1 status. This method allows discrete specification of time and the uncertainty of the exact time at which an event occurs within a discrete interval of time. A proportional hazard model for such data can be fitted with software for binary data and a complementary log-log link for customary interpretation of the covariate effect as estimated HRs in a Cox proportional hazards model [18]. All individuals were followed from the age of 18 until the first relationship, December 31, 2015, emigration, or death, whichever occurred first, with age as the underlying time scale.

Effect modification was estimated according to three age groups (18-22 years, 23-32 years, and more than 33 years), gender, and birth year, one at a time, by adding interaction terms in a likelihood ratio test. The age ranges was chosen to cover the early (young) adulthood period (18-22 years), the adulthood period where most form their life structure (23-32 years) and the settling down adulthood period (more than 33 years).

A second model was fitted in which indicators of somatic and psychiatric comorbidity at entry were added to the initial model. In sensitivity analyses, differences between groups (individuals with NF1 vs. population comparisons) were tested with a slightly stricter definition of cohabitation: lasting $\geq 2$ years in order to assess the association between NF1 and longer-term relationships. For a nonparametric comparison of NF1 and population comparisons, survival curves were plotted to account for interval-censored data.

The study protocol was approved by the Danish Data Protection Agency (Record 2014-41-2935) and the Human Research Protection Office (HRPO) (A-18370.i) (June 2015/2016).

\section{Results}

We included 787 individuals with NF1 and 7787 population comparisons. Of these, 342 (43\%) individuals with NF1 and 4102 (53\%) without NF1 formed a marital or cohabiting relationship during follow-up. The median follow-up time
Table 1 Descriptive characteristics of 787 individuals registered with NF1 in Denmark in 1997-2015 and 7787 population comparisons matched on gender and year of birth.

\begin{tabular}{|c|c|c|c|}
\hline & NF1 & $\begin{array}{l}\text { Population } \\
\text { comparisons }\end{array}$ & $P$ value \\
\hline & $n=787$ & $n=7787$ & \\
\hline Gender & & & 0.999 \\
\hline Male & $385(49 \%)$ & $3815(49 \%)$ & \\
\hline Female & $402(51 \%)$ & $3972(51 \%)$ & \\
\hline Birth year & & & 0.999 \\
\hline $1971-1975$ & $148(19 \%)$ & $1461(19 \%)$ & \\
\hline 1976-1980 & $144(18 \%)$ & $1430(18 \%)$ & \\
\hline 1981-1986 & $152(19 \%)$ & $1515(20 \%)$ & \\
\hline 1987-1992 & $178(23 \%)$ & $1776(23 \%)$ & \\
\hline 1993-1997 & $165(21 \%)$ & $1605(21 \%)$ & \\
\hline \multicolumn{4}{|l|}{$\begin{array}{l}\text { Somatic diseases at time } \\
\text { of entry }\end{array}$} \\
\hline Cancer $^{\mathrm{a}}$ & $68(9 \%)$ & $12(0.2 \%)$ & $<0.001$ \\
\hline $\begin{array}{l}\text { Diseases of bone, joints } \\
\text { and soft tissue } e^{b}\end{array}$ & $76(10 \%)$ & $159(2 \%)$ & $<0.001$ \\
\hline $\begin{array}{l}\text { Diseases of nervous } \\
\text { system }^{c}\end{array}$ & $130(17 \%)$ & $371(5 \%)$ & $<0.001$ \\
\hline $\begin{array}{l}\text { Diseases of circulatory } \\
\text { system }^{\mathrm{d}}\end{array}$ & $23(3 \%)$ & $40(1 \%)$ & $<0.001$ \\
\hline Other somatic diseases ${ }^{\mathrm{e}}$ & $465(59 \%)$ & $2722(35 \%)$ & $<0.001$ \\
\hline \multicolumn{4}{|l|}{$\begin{array}{l}\text { Psychiatric disorders at } \\
\text { time of entry }\end{array}$} \\
\hline Depression $^{\mathrm{f}}$ & $5(1 \%)$ & $40(1 \%)$ & 0.848 \\
\hline Anxiety ${ }^{g}$ & $5(1 \%)$ & $50(1 \%)$ & 1 \\
\hline $\begin{array}{l}\text { Other psychiatric } \\
\text { disorders }{ }^{\mathrm{h}}\end{array}$ & $123(16 \%)$ & $461(6 \%)$ & $<0.001$ \\
\hline
\end{tabular}

Percentages are rounded to whole numbers.

${ }^{\mathrm{a} C}$ Cancer; malignant (ICD-10): C00-C43, C46-C96, B21.0, D30.1-D30.9, D32-D33, D35.2-D35.4, D41.4, D42-D43, and D44.3-D44.5, benign (ICD-8): 210-221, 223.0, 224, 225.5-225.6, 226.0-226.1, and 226.4-228.

${ }^{\mathrm{b}}$ Diseases of bone, joints and soft tissue (ICD-8): 720-738, 274, and 710-718.

${ }^{\mathrm{c} D i s e a s e s}$ of nervous system (ICD-8): 290, 320-324, 340-342, 344-347, 349-358, and 360-389.

${ }^{\mathrm{d}}$ Diseases of circulatory system (ICD-8): 390-398, 400-404, 410-414, 420-438, 440-448, and 450-458.

${ }^{\mathrm{e}}$ Other somatic diseases (ICD-8): 000-136, 240-242, 244-269, 275.4-276, 278-279, 606, 626-628, 280-281, 283-289, 460-519, 520-543, 550-577, 580-605, 607-625, 629, 700-709, 680-686, 690-698, 274, 710-718, and 720-738.

${ }^{\mathrm{f}}$ Depression (ICD-10): F32.

${ }^{\mathrm{g}}$ Anxiety (ICD-10): F41.

${ }^{\mathrm{h}}$ Other psychiatric disorders (ICD-10): F01-99, excluding F32, F41, F84, and F90.

was 9.9 years (range $0-27$ years). Individuals with NF1 had significantly more somatic comorbid disorders than population comparisons (Table 1). 


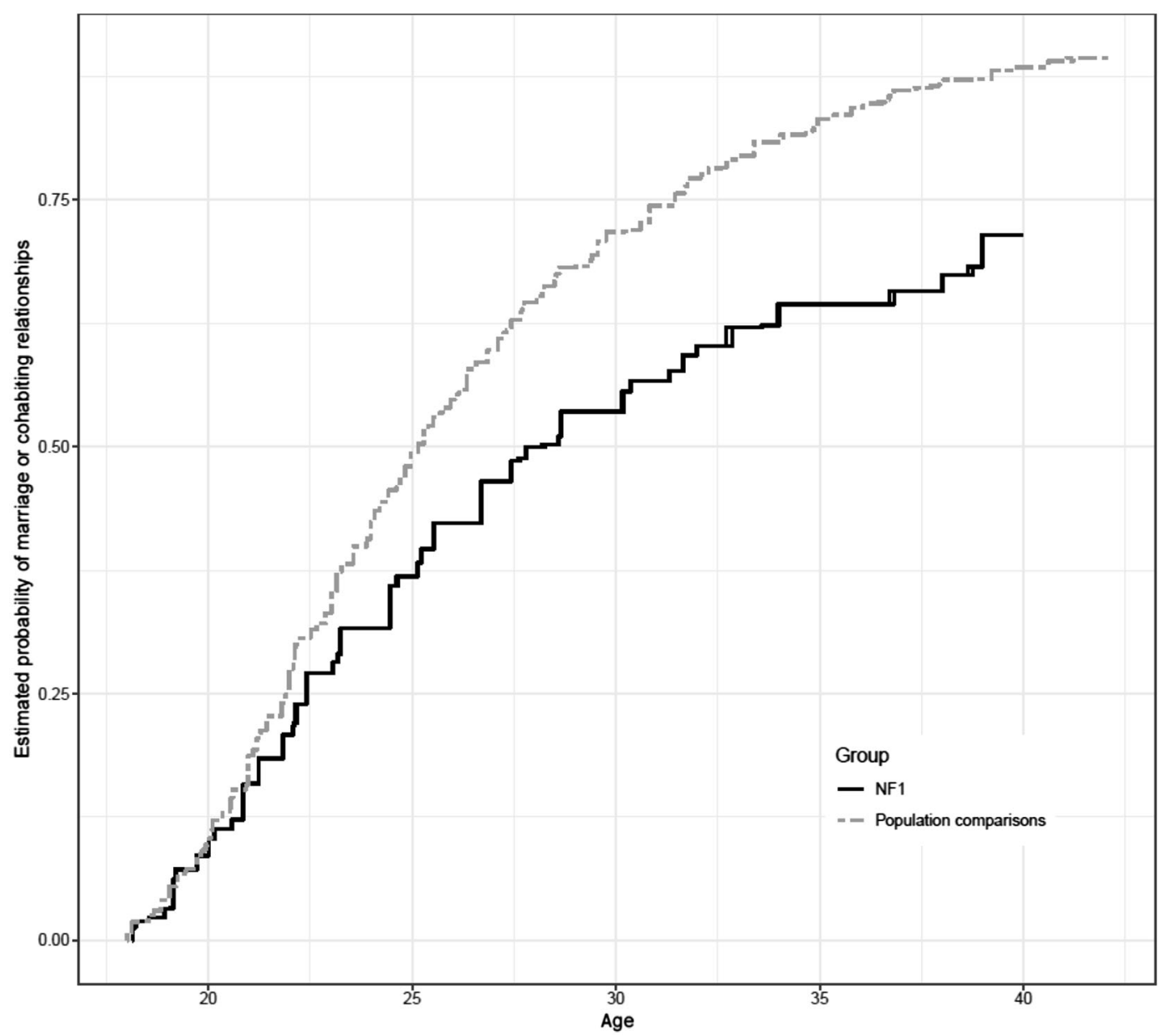

Fig. 1 Estimated probability of forming a first marriage or cohabiting relationship among Danish individuals with NF1 and matched population comparisons, by age. The cumulative probability

Overall, individuals with NF1 had a significantly lower overall hazard of forming a relationship than population comparisons ( $\mathrm{HR}=0.65$; 95\% CI: $0.58-0.73$ ) after adjustment for gender and birth year. Additional adjustment for somatic and psychiatric morbidity at the time of inclusion did not change the results $(\mathrm{HR}=0.64 ; 95 \% \mathrm{CI}: 0.57-0.71)$. Similar results were found in sensitivity analyses restricted to relationships lasting $\geq 2$ years (HR $0.66 ; 95 \% \mathrm{CI}$ : $0.58-0.75)$. Half of the individuals with NF1 had formed their first relationship by approximately 29 years of age, whereas population comparisons had done so by the age of 25 (Fig. 1).

The association between NF1 and cohabitation status was modified by attained age, with HR of 0.40 (95\% CI: $0.25-0.63$ ) for individuals $\geq 33$ years and HR 0.82 (95\% CI: $0.70-0.96$ ) for individuals aged $18-22$ years, indicating that the oldest individuals with NF1 had the lowest probability of marriage or cohabitation ( $P$ for interaction $<0.001$ ) (Table 2). Neither gender nor birth year significantly modified the association. over age of forming a marital or cohabiting relationship. The black solid line is the probability in the NF1 group and the grey dashed line is the population comparisons group.

Of the 4444 individuals who had formed a relationship during follow-up, we followed 342 individuals with NF1 and 4102 population comparisons until they separated. After adjustment for gender and birth year, no significant difference in couples with a NF1 individual was seen for ending a relationship (HR 1.00, 95\% CI: 0.86-1.16). The results did not change after additional adjustment for somatic and psychiatric morbidity (HR 0.93, 95\% CI: 0.80-1.09). Similar results were found in sensitivity analyses restricted to relationships lasting $\geq 2$ years (HR 0.91, 95\% CI: 0.76-0.90), and none of the added interactions was statistically significant, indicating that the hazard of ending a first longer relationship did not differ by age, gender, or birth year (data not shown).

\section{Discussion}

In this large population-based cohort study of the impact of NF1 on beginning and ending marital or cohabiting 
Table 2 Modification of the association between NF1 and forming a first marriage or cohabiting relationship among 787 individuals with NF1 compared to 7787 population comparisons matched on gender and year of birth.

\begin{tabular}{lllll}
\hline Effect & HR & $95 \%$ CI & $P$ value & $P$ value for interaction \\
\hline $\begin{array}{l}\text { By age } \\
\text { 18-22 years }\end{array}$ & 0.82 & $(0.70-0.96)$ & 0.01 & $<0.001$ \\
23-32 years & 0.57 & $(0.49-0.68)$ & $<0.001$ & \\
33 or older & 0.40 & $(0.25-0.63)$ & $<0.001$ & \\
By gender & & & & \\
Men & 0.59 & $(0.50-0.70)$ & $<0.001$ & 0.1 \\
Women & 0.70 & $(0.60-0.80)$ & $<0.001$ & \\
By birth year & & & & \\
1971-1975 & 0.68 & $(0.56-0.83)$ & $<0.001$ & 0.5 \\
1976-1980 & 0.64 & $(0.51-0.79)$ & $<0.001$ & \\
1981-1986 & 0.72 & $(0.58-0.90)$ & 0.004 & \\
1987-1992 & 0.58 & $(0.44-0.76)$ & $<0.001$ & \\
1993-2000 & 0.42 & $(0.20-0.89)$ & 0.02 & \\
\hline
\end{tabular}

relationships as documented by national registries, we found that individuals with NF1 had a significantly lower HR for engaging in a marriage or cohabiting relationship and were older when they entered their first relationship than population comparisons. The probability of marriage or cohabitation was lowest for the oldest individuals with NF1 compared to the youngest. Once in a relationship, however, couples with NF1 individuals were not at greater risk of ending it than population comparisons. Our findings illustrate the potential social consequences of this lifelong condition, which can have a huge impact on functioning, health-related quality of life, and daily life [7, 19]. Potential explanations for our study results could include reduced self-confidence especially related to body image as many individuals with NF1 experience fibromas during late adolescence and in adulthood [8,9]; reduced social cognition which may limit the understanding of social situations and social cues and also the skills required to navigate in relationships with others [20]; fatigue and lack of energy to engage in social activities [21]; reduced opportunities to meet others if education and work life is limited [12]; and uncertainty about prognosis and severity and the risk of passing on NF1 to the next generation may influence the decision not to have children which again has the potential to affect partnering and planning of family life [7].

The strengths of this first population-based study of marital or cohabiting relationships in individuals with NF1, include the nationwide cohort design, the large study population, annually updated information on cohabitation and marriage, and population-based comparisons with complete follow-up. The data were extracted from high- quality Danish population-based registries with data collected independently of the study hypotheses.

A limitation of this study is that only individuals hospitalized for NF1 were included in the study cohort, eliminating individuals with NF1 diagnosed in the primary health care system or with problems not severe enough for hospitalization. This limitation may reduce the generalizability of our results, as only individuals with more severe disease manifestations was included in the study. However, evidence whether or not disease severity may influence the individual's ability to engage in marriage or cohabitation is lacking. Previous studies have shown that the cosmetic visibility of NF1 may raise concern about appearance and greater sexual, body, and social self-consciousness [22, 23], which may be associated with lower self-esteem and more loneliness [22]. Conversely, qualitative studies indicate that mildly affected adults with NF1 have many of the same concerns as those more severely affected [9, 23]. Further, disease severity was not associated with social functioning in 62 individuals with NF1 and 39 unaffected adults on the Social Performance Survey Schedule [5]. A study of 49 individuals with NF1 found no difference in the self-esteem of those with NF1 who considered that their disease affected their appearance and those who did not, and it was suggested the cosmetic effects of NF1 may not be the cause of lower self-esteem but rather the cognitive dysfunction [8]. In our study, we adjusted for somatic and psychiatric morbidity at study entry, including ADHD and autism spectrum disorder, therefore partially taking into consideration the potential harmful cognitive effect of NF1 on forming marital or cohabiting relationships. Adjustment for these variables did not, however, change our results. Nonetheless, no firm conclusion can be drawn about the effect of disease severity in individuals with NF1 on their ability to form relationships in this register-based study, and more studies should be conducted with data from both registries and questionnaires.

Other potential limitations include the fact that information on beginning and ending marital or cohabiting relationships is registered only once a year, and any inaccuracy in time intervals might have had a small effect on the estimates. Further, although the definition of cohabitation in the Danish Household and Family Statistics is robust, some couples who met the definition but were not intimate could have been misclassified. Furthermore, we excluded couples who were institutionalized. In this study, we had no information of relationships outside marriage or cohabitation. It is likely that individuals with NF1 may have relationships without being married or cohabiting, and thus we underestimate the absolute number of persons who are in a relationship. However, we have no reason to believe that this affects the two groups compared differently. 


\section{Conclusions}

The ability to form and maintain marital or cohabiting relationships in adulthood plays an important role in health and well-being and is the basis for creating a supportive family life. In this large population-based cohort study on the impact of NF1 on forming and ending marital or cohabiting relationships, individuals with NF1 were less likely to engage in relationships than population comparisons and were older when they formed their first relationship. Once a marital or cohabiting relationship is established, however, couples with a NF1 individual are not at greater risk of ending the relationship than population comparisons. Health care professionals should take social difficulties into consideration when counseling and caring for people with NF1.

Acknowledgements The study was supported by a grant from the US Army Medical Research and Material Command (award number W81XWH-14-1-0054).

\section{Compliance with ethical standards}

Conflict of interest The authors declare that they have no conflict of interest.

Publisher's note Springer Nature remains neutral with regard to jurisdictional claims in published maps and institutional affiliations.

\section{References}

1. Stewart DR, Korf BR, Nathanson KL, Stevenson DA, Yohay K. Care of adults with neurofibromatosis type 1: a clinical practice resource of the American College of Medical Genetics and Genomics (ACMG). Genet Med. 2018;20:671-82.

2. Pride NA, Korgaonkar MS, Barton B, Payne JM, Vucic S, North $\mathrm{KN}$. The genetic and neuroanatomical basis of social dysfunction: lessons from neurofibromatosis type 1. Hum Brain Mapp. 2014;35:2372-82.

3. Chisholm AK, Anderson VA, Pride NA, Malarbi S, North KN, Payne JM. Social function and autism spectrum disorder in children and adults with neurofibromatosis type 1: a systematic review and meta-analysis. Neuropsychol Rev. 2018;28:317-40.

4. Ferner RE. Neurofibromatosis 1 and neurofibromatosis 2: a twenty first century perspective. Lancet Neurol. 2007;6:340-51.

5. Pride NA, Crawford H, Payne JM, North KN. Social functioning in adults with neurofibromatosis type 1. Res Dev Disabil. 2013;34:3393-9.

6. Ejerskov C, Lasgaard M, Ostergaard JR. Teenagers and young adults with neurofibromatosis type 1 are more likely to experience loneliness than siblings without the illness. Acta Paediatr. 2015;104:604-9.

7. Ablon J. Gender response to neurofibromatosis 1. Soc Sci Med. 1996;42:99-109.

8. Rosnau K, Hashmi SS, Northrup H, Slopis J, Noblin S, Ashfaq M. Knowledge and self-esteem of individuals with neurofibromatosis type 1 (NF1). J Genet Couns. 2017;26:620-7.

9. Hummelvoll G, Antonsen KM. Young adults' experience of living with neurofibromatosis type 1. J Genet Couns. 2013;22: 188-99.

10. Wang DL, Smith KB, Esparza S, Leigh FA, Muzikansky A, Park $\mathrm{ER}$, et al. Emotional functioning of patients with neurofibromatosis tumor suppressor syndrome. Genet Med. 2012;14:977-82.

11. Meier A, Allen G. Intimate relationship development during the transition to adulthood: differences by social class. New Dir Child Adolesc Dev. 2008;119:25-39.

12. Doser K, Kenborg L, Andersen EW, Bidstrup PE, Kroyer A, Hove $\mathrm{H}$, et al. Educational delay and attainment in persons with neurofibromatosis 1 in Denmark. Eur J Hum Genet. 2019;27:857-68.

13. National Institutes of Health Consensus Development Conference Statement: neurofibromatosis. Bethesda MD, USA, July 13-15, 1987. Neurofibromatosis. 1988;1:172-8.

14. Lynge E, Sandegaard JL, Rebolj M. The Danish National Patient Register. Scand J Public Health 2011;39(7 Suppl):30-3.

15. Pedersen CB. The Danish Civil Registration System. Scand J Public Health 2011;39(7 Suppl):22-5.

16. StatisticsDenmark https://www.dst.dk/en/Statistik/dokumentation/ documentationofstatistics/households--families-and-children; 2019.

17. Mors O, Perto GP, Mortensen PB. The Danish Psychiatric Central Research Register. Scand J Public Health 2011;39(7 Suppl):54-7.

18. Hosmer DW, Lemeshow S, May S. Applied Survival Analysis: Regression Modeling of Time-to-Event Data. Hoboken, New Jersey: John Wiley \& Sons, Inc; 2000.

19. Garwood MM, Bernacki JM, Fine KM, Hainsworth KR, Davies WH, Klein-Tasman BP. Physical, cognitive, and psychosocial predictors of functional disability and health-related quality of life in adolescents with neurofibromatosis-1. Pain Res Treat. 2012;2012:975364.

20. Allen T, Willard VW, Anderson LM, Hardy KK, Bonner MJ. Social functioning and facial expression recognition in children with neurofibromatosis type 1 . J Intellect Disabil Res. 2016;60:282-93.

21. Vassallo G, Mughal Z, Robinson L, Weisberg D, Roberts SA, Hupton E, et al. Perceived fatigue in children and young adults with neurofibromatosis type 1. J Paediatr Child Health. https://doi. org/10.1111/jpc.14764; 2020 [Epub ahead of print].

22. Smith KB, Wang DL, Plotkin SR, Park ER. Appearance concerns among women with neurofibromatosis: examining sexual/ bodily and social self-consciousness. Psychooncology 2013;22: 2711-9.

23. Crawford HA, Barton B, Wilson MJ, Berman Y, McKelveyMartin VJ, Morrison PJ, et al. The impact of neurofibromatosis type 1 on the health and wellbeing of Australian adults. J Genet Couns. 2015;24:931-44. 